Discussiones Mathematicae

Graph Theory 35 (2015) 355-363

doi:10.7151/dmgt.1790

\title{
EIGENVALUE CONDITIONS FOR INDUCED SUBGRAPHS
}

\author{
Jochen Harant $^{1}$, Julia Niebling ${ }^{1}$ \\ AND \\ Sebastian Richter $^{2}$ \\ ${ }^{1}$ Ilmenau University of Technology \\ Department of Mathematics, Germany \\ ${ }^{2}$ Chemnitz University of Technology \\ Department of Mathematics, Germany \\ e-mail: jochen.harant@tu-ilmenau.de \\ julia.niebling@tu-ilmenau.de \\ sebastian.richter@mathematik.tu-chemnitz.de
}

\begin{abstract}
Necessary conditions for an undirected graph $G$ to contain a graph $H$ as induced subgraph involving the smallest ordinary or the largest normalized Laplacian eigenvalue of $G$ are presented.
\end{abstract}

Keywords: induced subgraph, eigenvalue.

2010 Mathematics Subject Classification: 05C50.

\section{REFERENCES}

[1] W.N. Anderson Jr. and T.D. Morley, Eigenvalues of the Laplacian of a graph, Linear Multilinear Algebra 18 (1985) 141-145. doi:10.1080/03081088508817681

[2] D.P. Bertsekas, Nonlinear Programming: Second Edition (Athena Scientific, Belmont, Massachusetts 1999) page 278, Proposition 3.1.1.

[3] B. Bollobás and V. Nikiforov, Graphs and Hermitian matrices: eigenvalue interlacing, Discrete Math. 289 (2004) 119-127.

doi:10.1016/j.disc.2004.07.011

[4] A.E. Brouwer and W.H. Haemers, Spectra of Graphs (Springer Verlag, 2011). 
[5] S. Butler, Interlacing for weighted graphs using the normalized Laplacian, Electron. J. Linear Algebra 16 (2007) 90-98.

[6] F.R.K. Chung, Laplacian of graphs and Cheeger's inequalities, in: Combinatorics, Paul Erdôs is Eighty, János Bolyai Math. Soc., Budapest Vol. 2, 1996, 157-172.

[7] D.M. Cvetković, M. Doob and H. Sachs, Spectra of Graphs: Theory and Applications (Johann Abrosius Barth Verlag, Heidelberg-Leipzig, 1995).

[8] R. Diestel, Graph Theory (Springer, Graduate Texts in Mathematics, 173, 2000).

[9] W.H. Haemers, Interlacing eigenvalues and graphs, Linear Algebra Appl. 1995 (227-228) 593-616.

doi:10.1016/0024-3795(95)00199-2

[10] F.J. Hall, The Adjacency Matrix, Standard Laplacian, and Normalized Laplacian, and Some Eigenvalue Interlacing Results, Department of Mathematics and Statistics Georgia State University Atlanta, GA 30303.

http://www2.cs.cas.cz/semincm/lectures/2010-04-13-Hall.pdf

[11] J. Harant and S. Richter, A new eigenvalue bound for independent sets, Discrete Math. accepted.

http://www.tu-chemnitz.de/mathematik/preprint/2014/PREPRINT_08.pdf.

[12] V.S. Shigehalli and V.M. Shettar, Spectral techniques using normalized adjacency matrices for graph matching, Int. J. Comput. Sci. Math. 2 (2011) 371-378.

[13] Xiao-Dong Zhang, The Laplacian eigenvalues of graphs: a survey. arXiv:1111.2897v1 [math.CO] 12Nov2011

[14] R. Zurmühl, Matrizen (Springer 1950) 152-158. doi:10.1007/978-3-642-53289-4 16

Received 21 January 2014

Revised 10 June 2014

Accepted 11 June 2014 